\title{
A PAEDIATRIC DEATH DUE TO MENINGITIS-AN AUTOPSY DIAGNO- SIS OF A RARE COMPLICATION OF INFLUENZA B INFECTION
}

\author{
Borukgama N. ${ }^{1}$ and Hulathduwa S. R. ${ }^{2}$ 厌 (1) \\ ${ }^{1}$ Registrar in Forensic Medicine, Department of Forensic Medicine, Faulty of Medical Sciences, University of Sri Jayawar- \\ denepure, Nugegoda, Sri Lanka \\ ${ }^{2}$ Senior Lecturer/Consultant Forensic Pathologist, Department of Forensic Medicine, Faulty of Medical Sciences, Univer- \\ sity of Sri Jayawardenepure, Nugegoda, Sri Lanka
}
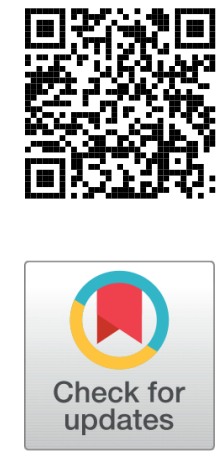

Received 3 April 2021

Accepted 18 April 2021

Published 30 April 2021

Corresponding Author

Hulathduwa S. R., sanjayarh@yah

oo.co.uk, sanjaya@sjp.ac.lk

DOI $10.29121 /$

granthaalayah.v9.i4.2021.3905

Funding: This research received no specific grant from any funding agency in the public, commercial, or not-for-profit sectors.

Copyright: (C) 2021 The Author(s). This is an open access article distributed under the terms of the Creative Commons Attribution License, which permits unrestricted use, distribution, and reproduction in any medium, provided the original author and source are credited.

OPEN ACCESS

\section{ABSTRACT}

Influenza virus belongs to a group of single-stranded RNA viruses in the family Orthomyxoviridae. They are subdivided into four types as A, B, C and D. As Influenza B is considered a milder form of infection not known to be causing pandemics compared to Influenza $A$, it is equally a less researched topic than Influenza A. However, few studies have shown that the mortality associated with pediatric Influenza B infection is equal to or sometimes even greater than the mortality associated with Influenza A. Complications of Influenza A or B virus infection include pneumonia, myositis, myocarditis and encephalitis/encephalopathy. Influenza-associated encephalitis/encephalopathy (IAE) is a rare complication of Influenza infection which is commoner following infection with type A. This case elaborates a death of a three-year-old child due to meningoencephalitis following Influenza Type B infection.

Keywords: InfluenzaAssociated Encephalopathy, Paediatric Deaths, Seizure, Cerebral Oedema

\section{CASE HISTORY}

A previously well three-year-old boy presented to the emergency treatment unit of a tertiary care hospital with a seizure attack following fever and vomiting of one-day duration. He has had an altered behavior before the seizure attack. He had no history of previous febrile convulsions. Though seizures have subsided soon after admission, the child was persistently drowsy and had a high fever with persistent tachycardia. Basic investigations showed a lymphocytosis and elevated liver enzymes. Despite intensive care, the child died within 12 hours of admission. As the cause of death was unknown, the death has been referred to the judicial medical officer to be subjected to a judicial autopsy following an inquest. 
The body was that of a male child with an adequate growth for the age and a good level of nutrition without any dysmorphic features. There were no external or internal injuries. The brain was $1510 \mathrm{~g}$ in weight with gross cerebral oedema and marked vascular congestion. There were softening and liquefactive necrosis in periventricular areas and thalamic area of the cerebrum. The cerebellum also showed marked oedema. There were no intracerebral or brainstem hemorrhages. Localized areas of pus collection or abscess formation were not evident.
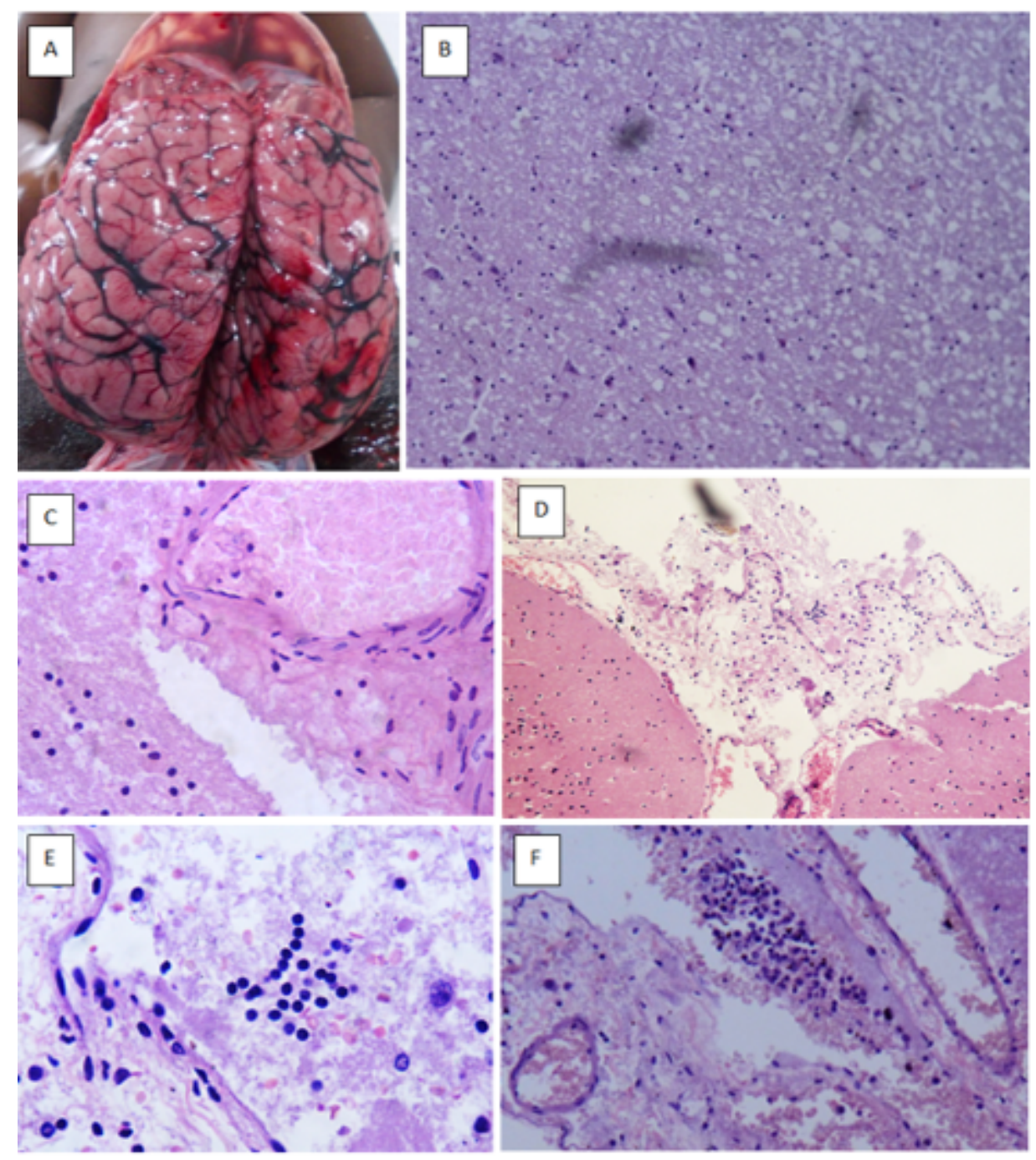

Figure 1 A: Macroscopic appearance of the brain during the autopsy. H\&E-stained sections of the brain showing B: cerebral oedema. C: Showing vascular congestion. D-F: H\&E-stained section of brain with leptomeninges showing lymphocytic infiltration with extravasated red cells

Microscopy of the brain showed significant interstitial oedema with very few lymphocytic infiltrations. No perivascular cuffing was observed even though the cerebral vessels were congested. However, the leptomeninges showed inflammation with lymphocytic infiltration and vascular congestion with some extravasated red cells 
in the sub-arachnoid space. Respiratory system showed mild pulmonary oedema on macroscopy. The microscopy of the respiratory system showed interstitial inflammation with occasional lymphocytic infiltration.
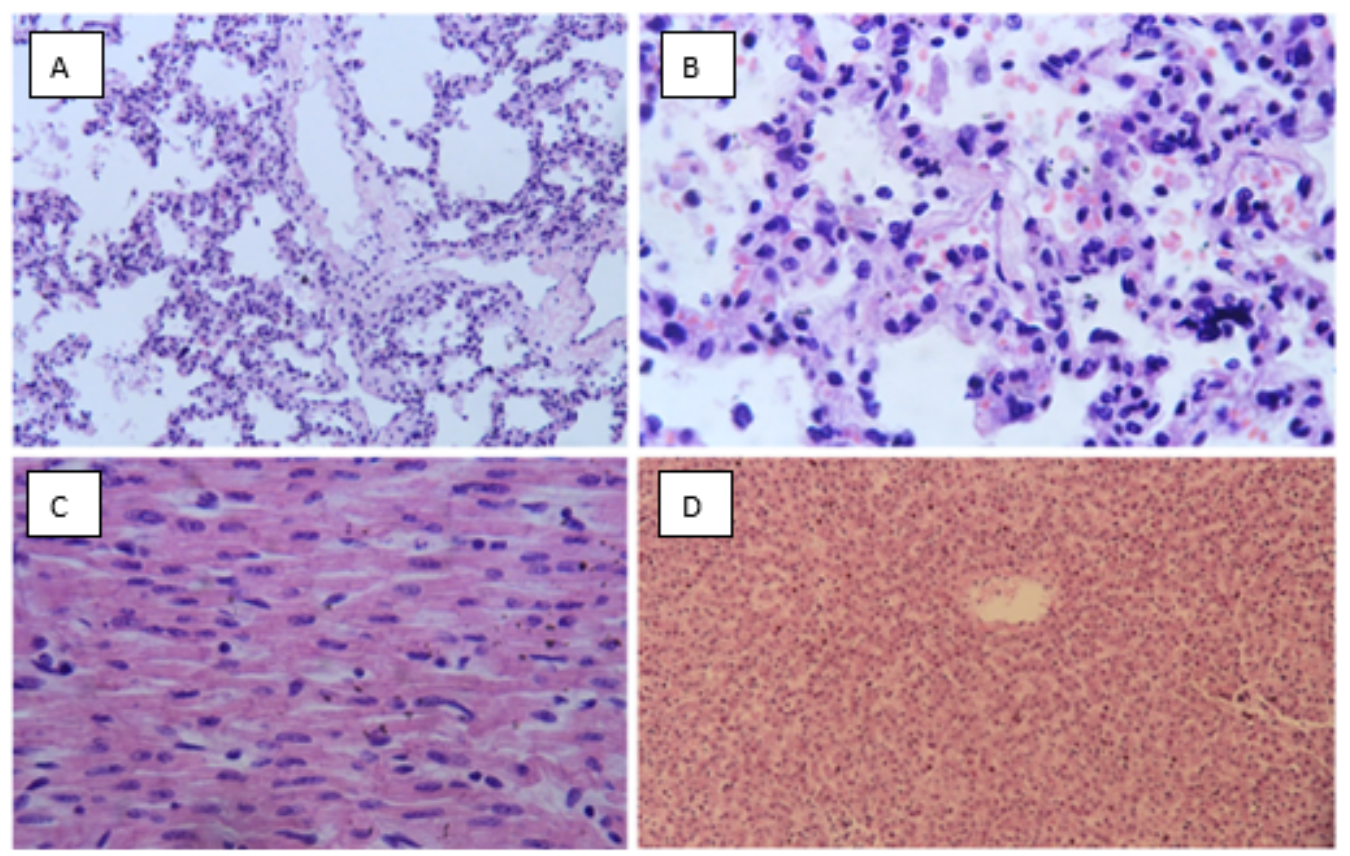

Figure 2 H\&E-stained sections of the lung A- (Low power) B- Interstitial inflammation with lymphocytes and red cell extravasation. C-H\&E-stained section of heart. D-H\&E-stained section of the liver.

Cardiovascular system examination did not reveal congenital or any other form of abnormality macroscopically and microscopically. Examination of the gastrointestinal, hepatobiliary and genitourinary systems were unremarkable. Patient's serum and nasopharyngeal swabs were taken and sent for virological studies including dengue and influenza. The nasopharyngeal swab showed molecular evidence of Influenza Type B specific RNA (Ribonucleic acid) on reverse transcription polymerase chain reaction (RT-PCR) testing.

\section{DISCUSSION}

Influenza virus belongs to a group of single-stranded RNA viruses in the family Orthomyxoviridae Newland et al. (2003). They are subdivided into four types as A, B, C and D Wolff and Veit (2021). As Influenza B is considered a milder form of infection not known to be causing pandemics compared to Influenza A, it is equally a less researched topic than Influenza A Tran et al. (n.d.). However, few studies have shown that the mortality associated with pediatric Influenza B infection is equal to or sometimes even greater than the mortality associated with Influenza A Tran et al. (n.d.). Complications of Influenza A or B virus infection include pneumonia, myositis, 
myocarditis and encephalitis/encephalopathy Newland et al. (2003). Encephalitis and meningo-encephalitis in childhood could be due to a variety of aetiologies including viruses, bacteria, mycobacteria, fungi, protozoa and immune mediated disorders Aneja and Sharma (2019). Influenza-associated encephalopathy or encephalitis (IAE) is a rare complication following influenza infections and it is a less common occurrence in influenza type B infections compared to infection with Influenza Type A. Influenza virus associated encephalopathy (IAE) is a disease of young children with a peak incidence between 6 and 18 months of age Morishima et al. (2002). It has mostly been reported from Japan and Taiwan though cases have been reported from Europe, North America and among other Caucasian children Kirton et al. (2005). The most severe category of IAE is acute necrotizing encephalopathy (ANE) which is commonly associated with long-term neurological sequelae and fatalities Howard et al. (2018).

Related literature states that IAE is a disorder with two phases. Usually on the first day the child will develop symptoms suggestive of influenza alone. Influenzaassociated encephalopathy develops later on the second or third day. Out of those who develop encephalopathy, approximately 33\% of the children will die (half from multi-organ failure and the other half from brain stem failure), another $33 \%$ will survive with neurological sequelae and cerebral atrophy and the remaining $33 \%$ will recover uneventfully. The above-mentioned outcome is expected only with the standard neuro-intensive care for a child with acute encephalopathy which will include ventilation, circulatory support, treatment and control of seizures, monitoring and treatment of raised intracranial pressure, treating with broad spectrum antibiotics, antivirals and often with steroids which may act as immune modulators. This child also had a similar clinical picture which reconfirms the findings in the literature Surtees (2006).

Common clinical presentation of encephalitis and meningo-encephalitis are fever, confusion, disorientation, coma and newly-onset seizures (excluding simple febrile seizures). This child had a rapid progression of the febrile illness with early deterioration of mental state and single episode of seizures. The child did not have any background of immunodeficiency, travel history, exposure to toxins or any drugs or recent vaccination. Furthermore, he did not have any prodromal illness or any respiratory symptoms during the course of his illness. No radiological investigations or cerebrospinal fluid analysis had been performed as clinical investigations in this case owing to the time-constraints due to very rapid deterioration of the clinical condition until the death resulted after the first few hours of admission.

The major macroscopic and microscopic findings during the autopsy examination were mostly based on the central nervous system. The cerebrum had marked oedema observed macroscopically as flattened gyri and narrowed sulci with a brain heavier than what is expected for the age of three years. However, signs of cerebral herniation were not observed in this case. In acute necrotizing encephalitis, a more severe form of IAE, symmetrical necrosis of the white and grey structures 
could be seen Mizuguchi et al. (1995), characterized by bilateral thalamic necrosis and petechiae with variable white matter oedema Achiriloaie et al. (2016). In this case under discussion, softening and liquefaction of periventricular white matter of the cerebrum, corpus callosum and surrounding white matter of the cerebellum were seen. However, the microscopic findings in this case were mostly observed in association with meninges. Perivascular infiltration of inflammatory cells or vasculopathies were not seen in this case. Additionally, there were features of interstitial inflammation with lymphocytic infiltrations observed in the lungs microscopically, although patient did not have significant symptoms. There were no other significant pathologies evident in other organ systems. The virological studies carried out on the nasal and throat swabs showed molecular evidence of Influenza Type B infection.

Considering the clinico-pathological evidence substantiated by virological evidence, the cause of death could be concluded as Meningoencephalitis associated with Influenza Type B infection. Probable Mechanism of death in this case could be due to electrical interference of the central nervous system secondary to the cerebral edema and cerebral hypoxia Wu et al. (2006).

\section{CONCLUSION}

This case highlights the importance of considering Influenza B infection as a serious condition in children specially when presented with neurological features as it can prove to be rapidly fatal. Furthermore, the importance of application of virological and histopathological studies as an important adjunct to macroscopic autopsy examinations should be appreciated by the autopsy pathologists.

\section{REFERENCES}

Achiriloaie, A., Michelson, D., Lei, L., Denham, L., Oberg, K., \& Raghavan, R. (2016). Acute Postviral Encephalopathy: Pathologicand Radiologic Correlation In An Atypical Case. Child Neurology Open, 1-16. 10.1177/2329048x16658845

Aneja, S., \& Sharma, S. (2019). Diagnosis and Management of Acute Encephalitis in Children. The Indian Journal of Pediatrics, 86(1), 70-75. Retrieved from https://dx.doi.org/10 .1007/s12098-018-2775-0 10.1007/s12098-018-2775-0

Howard, A., Uyeki, T. M., \& Fergie, J. (2018). Influenza-Associated Acute Necrotizing Encephalopathy in Siblings. Journal of the Pediatric Infectious Diseases Society, 7(3), e172-e177. Retrieved from https://dx.doi.org/10.1093/jpids/piy033 10.1093/jpids/ piy033

Kirton, K. A., Busche, C., Ross, E., \& Wirrell. (2005). Acute Necrotizing Encephalopathy In Caucasian Children: Two Cases And Review Of The Literature. J Child Neurol, 527-559.

Mizuguchi, M., Abe, J., Mikkaichi, K., Noma, S., Yoshida, K., Yamanaka, T., \& Kamoshita, S. (1995). Acute necrotising encephalopathy of childhood: a new syndrome presenting with multifocal, symmetric brain lesions. Journal of Neurology, Neurosurgery \& Psychiatry, 58(5), 555-561. Retrieved from https://dx.doi.org/10.1136/jnnp.58.5.555 10.1136/jnnp.58.5.555 
Morishima, T., Togashi, T., Yokota, S., Okuno, Y., Miyazaki, C., Tashiro, M., \& and, N. O. (2002). Encephalitis and Encephalopathy Associated with an Influenza Epidemic in Japan. Clinical Infectious Diseases, 35(5), 512-517. Retrieved from https://dx.doi.org/10.1086/ 341407 10.1086/341407

Newland, J. G., Romero, J. R., Varman, M., Drake, C., Holst, A., Safranek, T., \& Subbarao, K. (2003). Encephalitis Associated with Influenza B Virus Infection in 2 Children and a Review of the Literature. Clinical Infectious Diseases, 36(7), e87-e95. Retrieved from https://dx.doi.org/10.1086/368184 10.1086/368184

Surtees, R. (2006). Influenza virus associated encephalopathy. Archives of Disease in Childhood, 91, 455-456. Retrieved from https://dx.doi.org/10.1136/adc.2005.092890 10 $.1136 /$ adc.2005.092890

Tran, D., Vaudry, W., Moore, D., Ja, B., Sa, H., Dw, S., \& Al, E. (n.d.). Hospitalization For Influenza A Versus B. Pediatrics. Hospitalization For Influenza A Versus B. Pediatrics. Retrieved from Http://Dx.Doi.Org/10.1542/Peds.2015-4643)

Wolff, T., \& Veit, M. (2021). Encyclopedia Of Virology. Influenza B, C And D Viruses (Orthomyxoviridae). Encyclopedia Of Virology, 561-74.

Wu, S., Dj, S., \& Rs, F. (2006). Spitz And Fisher's Medicolegal Investigation Of Death. In Spitz And Fisher's Medicolegal Investigation Of Death: Guidelines For The Application of Pathology To Crime Investigation. Charles C. Thomas. 